International Mathematical Forum, Vol. 8, 2013, no. 35, 1723 - 1726

HIKARI Ltd, www.m-hikari.com

http://dx.doi.org/10.12988/imf.2013.39173

\title{
A Generalization of Van der Pol Equation
}

\author{
Ana-Magnolia Marin-Ramirez \\ Faculty of Exact and Natural Sciences \\ University of Cartagena, Sede Piedra de Bolivar \\ Avenue of Consulado, Cartagena de Indias \\ Bolivar, Colombia \\ amarinr@unicartagena.edu.co \\ Ruben-Dario Ortiz-Ortiz \\ Faculty of Exact and Natural Sciences \\ University of Cartagena, Sede Piedra de Bolivar \\ Avenue of Consulado, Cartagena de Indias \\ Bolivar, Colombia \\ rortizo@unicartagena.edu.co \\ Joel-Arturo Rodriguez-Ceballos \\ Morelia Institute of Technology, Morelia \\ Mexico \\ joel@ifm.umich.mx
}

Copyright (C) 2013 Ana-Magnolia Marin-Ramirez, Ruben-Dario Ortiz-Ortiz and JoelArturo Rodriguez-Ceballos. This is an open access article distributed under the Creative Commons Attribution License, which permits unrestricted use, distribution, and reproduction in any medium, provided the original work is properly cited.

\begin{abstract}
In this paper we make an analysis of a generalization of van der Pol equation without periodic orbits in a domain on the plane. We use a Gasull's result and Dulac's criterion.
\end{abstract}

Mathematics Subject Classification: 34A34

Keywords: Dulac functions, Quasilinear partial differential equations, Bendixon-Dulac criterion 


\section{Introduction}

It is important to make in differential equations the study of periodic orbits in the plane. Certain systems do not have limit cycles. It should be considered: Bendixson's criteria and critical points. (See $[4,5]$ ). In this paper we are interested in studying a generalization of a van der Pol equation that have a periodic orbit but in a circular domain of radius one and center in the origin there is no this limit cycle (See [1]). We use the criterion of Bendixson-Dulac (See [3]) and paper of Gasull (See [1]).

\section{Preliminary Notes}

Theorem 2.1. (Bendixson-Dulac criterion) (See [3]) Let $f_{1}\left(x_{1}, x_{2}\right), f_{2}\left(x_{1}, x_{2}\right)$ and $h\left(x_{1}, x_{2}\right)$ be functions $C^{1}$ in a simply connected domain $D \subset \mathbb{R}^{2}$ such that $\frac{\partial\left(f_{1} h\right)}{\partial x_{1}}+\frac{\partial\left(f_{2} h\right)}{\partial x_{2}}$ does not change sign in $D$ and vanishes at most on a set of measure zero. Then the system

$$
\left\{\begin{array}{l}
\dot{x}_{1}=f_{1}\left(x_{1}, x_{2}\right) \\
\dot{x}_{2}=f_{2}\left(x_{1}, x_{2}\right), \quad\left(x_{1}, x_{2}\right) \in D
\end{array}\right.
$$

does not have periodic orbits in $D$.

According to this criterion, to rule out the existence of periodic orbits of the system (1) in a simply connected region $D$, we need to find a function $h\left(x_{1}, x_{2}\right)$ that satisfies the conditions of the theorem of Bendixson-Dulac, such function $h$ is called a Dulac function. Our goal is to study a dynamical system on the plane that not have periodic orbits in a circular domain of radius one and center in the origin.

\section{Method to Obtain Dulac functions}

A Dulac function for the system (1) satisfies the equation

$$
f_{1} \frac{\partial h}{\partial x_{1}}+f_{2} \frac{\partial h}{\partial x_{2}}=h\left(c\left(x_{1}, x_{2}\right)-\left(\frac{\partial f_{1}}{\partial x_{1}}+\frac{\partial f_{2}}{\partial x_{2}}\right)\right)
$$

Theorem 3.1. (See [3]) For the system of differential equations (1), if (2) (for some function $c$ which does not change of sign and it vanishes only on a subset of measure zero) has a solution $h$ on $D$ such that $h$ does not change sign and vanishes only on a subset of measure zero, then $h$ is a Dulac function for (1) on $D$. 
Theorem 3.2. (See [1]) Assume that there exist a real number $s$ and an analytic function $h$ in $R^{2}$ such that

$$
f_{1} \frac{\partial h}{\partial x_{1}}+f_{2} \frac{\partial h}{\partial x_{2}}=h\left(c\left(x_{1}, x_{2}\right)-s\left(\frac{\partial f_{1}}{\partial x_{1}}+\frac{\partial f_{2}}{\partial x_{2}}\right)\right)
$$

does not change sign in an open region $W \subset R^{2}$ with regular boundary and vanishes only in a null measure Lebesgue set. Then the limit cycles of system (1) are either totally contained in $\mathfrak{h}_{0}:=\{h=0\}$, or do not intersect $\mathfrak{h}_{0}$. Moreover the number $N$ of limit cycles that do not intersect $\mathfrak{h}_{0}$ satisfies $N=$ 0 if $s=0$.

\section{Main Results}

These are the main results of the paper.

Theorem 4.1. Let $f\left(x_{1}, x_{2}\right)$ be a function $C^{1}$ in a simply connected domain $D=\{h \leq 0\} \subset \mathbb{R}^{2}$ where $h\left(x_{1}, x_{2}\right)=\psi\left(x_{1}\right)+a x_{2}^{2}+b x_{2}+c$ and $\psi\left(x_{1}\right)$ is a function $C^{1}$ in $\mathbb{R}, a, b, c \in \mathbb{R}$ with the following conditions $x_{2} \psi^{\prime}\left(x_{1}\right)-\left(x_{1}+\right.$ $\left.f\left(x_{1}, x_{2}\right) x_{2}\right)\left(2 a x_{2}+b\right)$ which does not change sign and it vanishes only in a null measure Lebesgue subset and $b^{2}-4 a\left(\psi\left(x_{1}\right)+c\right) \geq 0$. Then the system

$$
\left\{\begin{array}{l}
\dot{x}_{1}=x_{2} \\
\dot{x}_{2}=-x_{1}-f\left(x_{1}, x_{2}\right) x_{2}
\end{array}\right.
$$

does not have periodic orbits on $D$.

Proof. Applying Theorem 3.2 to (4) (this system has critical point on $x_{2}=$ $\left.x_{1}=0\right)$. From (3) we see function $f\left(x_{1}, x_{2}\right)$ and values of $s$ satisfy the equation

$$
x_{2} h_{x_{1}}-\left(x_{1}+f x_{2}\right) h_{x_{2}}=h\left(c\left(x_{1}, x_{2}\right)+s\left(\frac{\partial f}{\partial x_{2}}\left(x_{1}, x_{2}\right) x_{2}+f\right)\right)
$$

for some $h, c\left(x_{1}, x_{2}\right)$ with $h c$ does not change of sign (except in a set of measure $0)$. Obviously $h$ is a Dulac function in certain cases. We propose (instead of try to solve equation (5)) the function $h=\psi\left(x_{1}\right)+a x_{2}^{2}+b x_{2}+c$ for adequate $\psi$ such that $h$ has a closed curve of level 0 . When $h=0$, we have $x_{2}=$ $\frac{-b \pm \sqrt{b^{2}-4 a\left(\psi\left(x_{1}\right)+c\right)}}{2 a}$. Then $b^{2}-4 a\left(\psi\left(x_{1}\right)+c\right) \geq 0$. We try to find the domain for which the system does not have periodic orbits. We have $h_{x_{1}}=\psi^{\prime}\left(x_{1}\right), \quad h_{x_{2}}=$ $2 a x_{2}+b$. So we have

$$
x_{2} \psi^{\prime}\left(x_{1}\right)-\left(x_{1}+f x_{2}\right)\left(2 a x_{2}+b\right)-\operatorname{sh}\left(\frac{\partial f}{\partial x_{2}}\left(x_{1}, x_{2}\right) x_{2}+f\left(x_{1}, x_{2}\right)\right)
$$


which does not change sign and it vanishes only in a null measure Lebesgue subset. Making $s=0$ (this system would not have periodic orbits inside the domain with boundary $h=0)$ we get that $x_{2} \psi^{\prime}\left(x_{1}\right)-\left(x_{1}+f\left(x_{1}, x_{2}\right) x_{2}\right)\left(2 a x_{2}+b\right)$ which does not change sign and it vanishes only in a null measure Lebesgue subset.

Example 4.2. Consider the system

$$
\left\{\begin{array}{l}
\dot{x}_{1}=x_{2} \\
\dot{x}_{2}=-x_{1}+\left(1-x_{1}^{2}\right)\left(1+x_{2}^{2}\right) x_{2} .
\end{array}\right.
$$

Taking $h\left(x_{1}, x_{2}\right)=x_{1}^{2}+x_{2}^{2}-1$ we obtain that the associated equation given in (3) with $s=0$ is $h c\left(x_{1}, x_{2}\right)=2 x_{2}^{2}\left(1-x_{1}^{2}\right)\left(1+x_{2}^{2}\right)$. So, this function does not change sign and it is zero only at $x_{2}=0, x_{1}= \pm 1$. The system does not contain periodic orbits on $D=\left\{x_{1}^{2}+x_{2}^{2} \leq 1\right\}$. By (6), we have $\ddot{x}_{1}+\left(x_{1}^{2}-\right.$ 1) $\left(\dot{x}_{1}^{2}+1\right) \dot{x}_{1}+x_{1}=0$. This equation is generalized by $\ddot{x}_{1}+f\left(x_{1}, \dot{x}_{1}\right) \dot{x}_{1}+x_{1}=0$. The last equation was studied in [2].

Acknowledgements. The authors express their deep gratitude to CONACYTMéxico, Programa de Mejoramiento del Profesorado (PROMEP)-México and Universidad de Cartagena for financial support.

\section{References}

[1] A. Gasull and H. Giacomini, Some applications of the extended Bendixson-Dulac theorem, (2013). http://arxiv.org/abs/1305.3402.

[2] J. Jiang, X. Wang and P. Yan, Analysis of global bifurcation for a class of systems of degree five, J. Math. Annal. Appl, 222 (1998), 305-318.

[3] O. Osuna and G. Villaseñor, On the Dulac Functions, Qual. Theory Dyn. Syst, 10 (1) (2011), 43-49.

[4] L. Perko, Differential Equations And Dynamical Systems, Springer, Berlin, 2006.

[5] L. Stephen, Dynamical systems with applications using MAPLE, Birkhäuser, Boston, 2001.

\section{Received: September 19, 2013}

